\title{
Ruggedising biomedical devices for field-testing in resource-constrained environments: Context, issues and solutions
}

\author{
Sarah Schopman, Kyle Kalchthaler, Ryan Mathern, Khanjan Mehta* and Peter Butler \\ Humanitarian Engineering and Social Entrepreneurship (HESE) Program; \\ Department of Bioengineering, The Pennsylvania State University, University Park, PA 16802, USA \\ *khanjan@engr.psu.edu
}

\begin{abstract}
Community Health Workers (CHWs) are community members who address primary health challenges through education, prevention, and awareness initiatives. CHWs conduct home visits, provide treatment for simple common illnesses, and offer health education on numerous topics including nutrition, child health, and family planning. Since they serve on the frontlines of healthcare in rural communities, ruggedised and low-cost biomedical devices could improve the efficiency and efficacy of their caregiving efforts. However, the vast majority of biomedical devices used in sub-Saharan Africa are designed by engineers in Western countries who are not familiar with the distinct physical, environmental, socio-cultural, and economic environment of the context for which they are designing. Systemic challenges include long distances, poor transportation, unreliable infrastructure, harsh climate, and limited operator education. Specifically, three sets of hurdles to the adoption, sustainability and usability of devices by the CHWs include vibrations and wire strain, dust and water penetration, and device usability. This article discusses the operational context of $\mathrm{CHWs}$ and then delves into the specific problems encountered, and practical solutions applied, during four years of field-testing ruggedised biomedical devices in rural Kenya.
\end{abstract}

KEYWORDS: Community health workers, ruggedised design, robustness, biomedical devices

\section{1}

\section{INTRODUCTION}

Poorly engineered medical devices contribute to the global health challenges of Sub-Saharan Africa and developing countries. Over $95 \%$ of the biomedical devices found in public hospitals in developing countries are imported, and five years after arrival, $96 \%$ of this donated equipment no longer functions (Malkin, 2007; Peña-Mohr, 1987). In sub-Saharan Africa, almost $70 \%$ of donated medical equipment is not in use due to lack of spare parts, insufficient means for maintenance, or simply because local personnel do not know how to use it (World Health Organisaiton, 2012). Engineering World Health $(\mathrm{EWH})$ surveyed 33 hospitals across 10 developing countries and found that $66 \%$ of medical equipment that was categoriSed as "broken", could in fact be repaired. EWH also determined that even though most medical device repair parts were available in developing countries, hospitals often chose not to spend scarce finances on replacing them because it was easier just to wait and request a new device.
The fundamental challenge is that the vast majority of biomedical devices being used in developing countries are designed by engineers in Western countries who are not familiar with the distinct physical, socio-cultural, and economic environment of sub-Saharan Africa (Dzombak et al, 2012).

Several educational programs focused on the design of biomedical devices for resource-constrained environments, such as the Duke Global Health Institute (2013) and the Center for Bioengineering Innovation and Design at Johns Hopkins University (n.d.), have emerged over the last few years. Design tools to assist engineering students and professionals to engage in context-driven design with a humancentric approach have been integrated into these educational and entrepreneurial regimens. One of the goals of these design programs is to ensure that the products are durable and robust and can withstand the harsh environment and non-specialised staff of developing countries. Their ruggedised devices must withstand climate demands such as high 
humidity, prolonged dust exposure, direct sunlight, extreme temperatures, drastic temperature changes, and exposure to water. Due to poor transportation infrastructure and rough handling of equipment, the devices need to be resistant to shock and have strain relief mechanisms. The longevity and affordability of these devices and their spare parts are essential due to high poverty levels and limited healthcare budgets that make expensive products and repairs impractical. Alongside the physical environment, engineers must possess an understanding of the healthcare landscape and user characteristics prior to engaging in design activities (Martin et al, 2008).

Engineers need to understand the implications of two major trends driving primary healthcare in developing countries. The burden of disease is growing disproportionately in different regions of the world, with developing countries moving toward a double burden of chronic and acute diseases. With changing lifestyles, chronic illnesses like hypertension, cancer, diabetes, and heart disease are gaining prominence and contributing to an increasing number of hospital visits and mortality. Due to the lack of doctors and nurses in developing countries, health professionals are simply not available to treat patients (Young et al, 2009; Lekoubou et al, 2010). Community Health Workers (CHWs) are trained community members who have stepped in to address primary health challenges through education, prevention and awareness activities. CHWs conduct home visits, provide treatment of simple and common illnesses, offer health and nutrition education, and provide support in maternal and child health, and family planning. They can contribute to the identification and subsequent referral of patients with illnesses such as Tuberculosis, HIV, malaria and acute respiratory disease. Depending on specific location and needs, CHWs perform different tasks ranging from record keeping and education to handing out basic medications and taking blood glucose measurements. All CHWs work with the primary goal of improving access to healthcare and decreasing morbidity and mortality. In essence, CHWs are at the forefront of healthcare in these communities and appropriately designed biomedical devices can improve their efficiency and efficacy (Buehler at al, 2013).

Towards this quest, the usability and robustness of the technology must be given as much prominence as its functionality and reliability (Foster, 2010). Compared to the relatively controlled environment of hospitals and clinics, the CHW's context of use is more dynamic and harsh. For example, during a CHW's home visit, the devices would be unpacked and set on a table, chair, or floor, while children or other family members will be in the vicinity and have access to the devices. The CHWs have limited education or relevant training and are limited in their ability to protect or maintain the devices. They travel with minimal medical equipment by foot or local transportation across the villages they serve. Electric power is unreliable and difficult to access, and batteries are expensive, making most electronic devices and appliances less attractive. At the same time, affordable computing and communication technologies like mobile phones (or cellular phones) present many opportunities for real-time coordination and data sharing.

Mobile phones and low-cost computers create opportunities for the design of low-cost biomedical devices based on virtual instrumentation. Commercial-off-the-shelf biomedical devices embed the primary sensor, signal conditioning, user display and (optional) networking support in the device itself. Virtualised devices consist of a sensor and the minimal essential hardware around it. A Data Acquisition (DAQ) device digitises the sensor output and the signal conditioning and user display are done on the computing device in software. Such devices can be designed to be modular, ruggedised, and affordable. Multiple biomedical devices can share a common data acquisition device. Hardware is more expensive and repairing the devices is unrealistic. Computer software is cheaper and easily upgradable through the cell phone itself. Some of the hardware can be manufactured locally using local materials and only the basic sensors, widely available and mostly inexpensive, have to be imported into the developing country. While all medical equipment is designed to work in a professional healthcare setting, the use of smart phones promotes success outside of a hospital and in the field where the CHWs are working. Hence there is a burning need for biomedical devices that will effectively serve the needs of the CHWs in the harsh East African context.

Undergraduate students in the biomedical engineering program at Pennsylvania State University, United States (or Penn State) have been designing low-cost ruggedised biomedical devices to be integrated into the Mashavu telemedicine system in rural East Africa (Fleishman et al, 2010). Mashavu is a telemedicine system currently deployed in Kenya to connect rural communities with the public primary care system. It has been researched and field-tested over the past six years by students and faculty from the Humanitarian Engineering and Social Entrepreneurship (HESE) Program at Penn State. Under the Mashavu framework, Community Health Workers (CHWs) operate kiosks where patients come to have their vital signs like temperature, weight, height and blood pressure measured. This data, along with a brief survey, is sent via GPRS to a secure website. Healthcare professionals, typically nurses, view this information and respond to the patient in less than 20 minutes. The reliability of this telemedicine system has been demonstrated to be comparable to similar systems in Western countries (Fleishman et al, 2010; Qin et al, n.d.). 


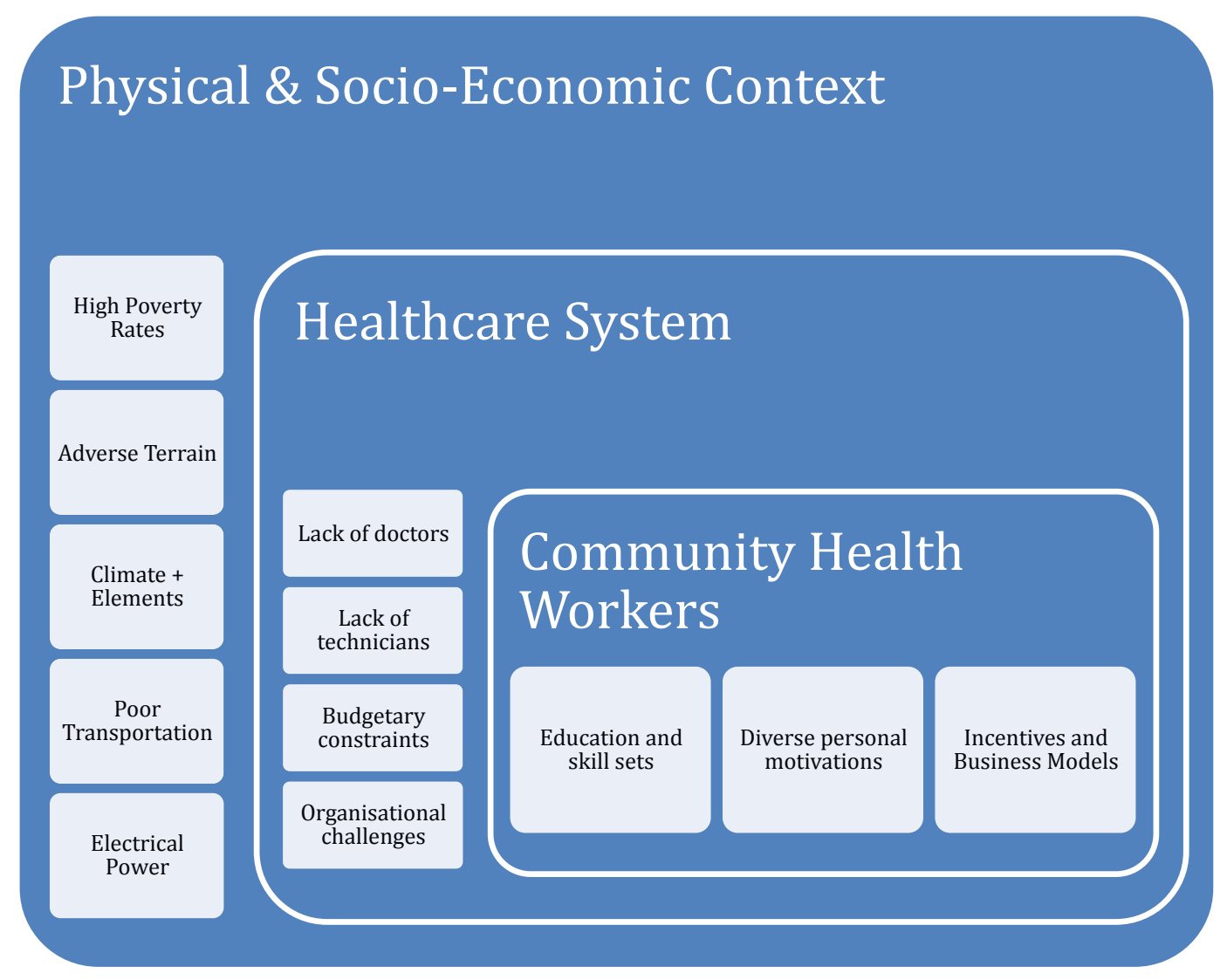

Figure 1: Context of use for biomedical devices in rural areas of developing countries.

The devices being developed for integration into Mashavu include a thermometer, adult weighing scale, baby weighing scale, pulse oximeter, blood pressure monitor, stethoscope, spirometer, electrocardiogram (EKG), dermascope, and ears, nose and throat (ENT) scope. All measurement information is transmitted from the biomedical instruments through a DAQ device to a LabVIEW program. Of the ten aforementioned devices, the blood pressure monitor, adult weighing scale, and thermometer have proven accurate through rigorous field-testing. The other seven devices have shown promise but need additional design improvements (Mathern et al, n.d.). During this beta testing phase, design prototypes have been tested alongside commercial biomedical devices, and found to be within acceptable accuracy and precision tolerances (Mathern et al, n.d.). Through successes and failures of field-testing iterations over the past four years, several lessons about appropriate ruggedisation techniques have been learned. As Mashavu continues to expand operations, all of these devices are undergoing further refinement to eventually prepare them for mass- manufacturing. The availability of these low-cost and ruggedised devices has the potential to accelerate the adoption of the Mashavu system in resource-constrained environments.

Through rigorous research and field-testing in the rural environment in which these devices operate, challenges and potential solutions related to the design of ruggedised biomedical devices have been identified and are presented in this paper. These problems range from practical difficulties, such as wire strain and vibration from daily transport, to the climate realities of rain and dust, to the operational challenges of limited training and designing culturally acceptable user experiences. The issues have been distilled into three key problem categories: transport vibrations and wire strain, dust and water exposure, and ease of use. Simple, practical, and inexpensive solutions have been developed to address these problems during the field-testing phases. While some of these solutions are just as relevant for the final product, the key assumption is that more sophisticated manufacturing processes like injection moulding will be used for mass-manufacturing these devices. This article first discusses the operational context of CHWs and then delves into the specific problems encountered, and practical solutions applied, during field-testing biomedical devices in a rural developing community context.

\section{CONTEXT OF USE FOR BIOMEDICAL DEVICES}

Systemic contextual issues that ultimately influence the design, field-testing, manufacturing and operation of biomedical devices by Community Health Workers (CHWs) are shown in Figure 1. 


\subsection{Physical and socio-economic context}

1. High poverty rates: Greater than half of the world's population, more than 3 billion people, lives on less than US\$2.50/day. Millions of these citizens, especially those living in rural areas, lack access to clean water, sufficient food, and adequate healthcare services (United Nations Development Program, 2007).

2. Adverse terrain: Rural roads are often unpaved and unattended. The air varies from very dusty in the dry seasons to very humid in rainy weather, leading to muddy terrain and uneven ground that must be traversed when travelling to remote villages. CHWs need to walk long distances to serve their communities.

3. Climate and elements: Several countries in SubSaharan Africa experience diverse and harsh climates. Throughout dry and rainy seasons, climates are characterised by high humidity, prolonged dust exposure, direct sunlight, extreme temperatures, drastic temperature changes, and exposure to water. Lack of reliable infrastructure complicates some of these factors and fails to provide adequate shelter and protection from environmental elements.

4. Poor transportation: Walking, bicycling or riding public minivans are the most common types of transportation for a CHW (Buehler at al, 2013). Exposure to adverse climate is inevitable since foot and bicycle travel are primary methods of travel. During transport, device design must account for overexposure to climate and terrain factors. For instance, wires may be pulled in unpacking, and devices will face shock loading on unpaved roads.

5. Electrical power: Reliable electricity is rare in subSaharan Africa. Batteries are often used to power radios, phones, TVs and other equipment in the field but they are expensive, heavy and difficult to transport. Their high costs and short lifespan further limit their use in rural areas.

\subsection{Healthcare system}

1. Lack of doctors: The doctor-to-patient ratio in developing countries can be as low as 1:50,000 (Doctors of the World, n.d.). The majority of these doctors are located in urban areas, though only $30 \%$ of the population in developing countries reside in these urban areas and can easily reach them (Chudi, 2010). Provider shortages are likely to persist, as many countries do not have the means to train adequate numbers of medical personnel.

2. Lack of technicians: In addition to a low doctorto-patient ratio, medical technicians with knowledge of how to maintain and repair biomedical devices are difficult to find. There are very few, if any, jobs for technicians and so devices are discarded even for minor easy-torepair faults.

3. Budgetary constraints: Developing countries have very limited budgets and funds to be spent on healthcare. In the United States an average of US\$7960 is spent per capita on healthcare each year. In contrast, only US\$75 is spent per capita in Kenya on an annual basis (World Health Organization, 2012). This presents a huge challenge in promoting healthcare in the rural areas where the cost of operations is higher due to the geographically dispersed population and poor infrastructure.

4. Organisational challenges: The hierarchical public healthcare system exists alongside private clinics and hospitals. Several non-profits and aid agencies also provide a variety of healthcare services, some of them focused on specific groups like pregnant women, children or elderly people. The rural populations often find it difficult to understand how these different resources are related to each other and whom they should approach for certain kinds of problems. The exact position and function of the CHWs in the healthcare system varies from place to place, further adding to the chaos in the system.

\subsection{Community health workers}

1. Education and skill sets: Rural communities are increasingly relying on CHWs to foster primary health. These individuals have varying levels of education ranging from basic indigenous skills to multi-week training programs through the local Ministries of Health, and may not be computer literate or have any advanced health education. As the primary operators of biomedical devices, the device design, user interface, and operational standards must accommodate CHWs' skill levels.

2. Diverse personal motivations: Community Health Workers currently operate on a volunteer basis in most countries. These locally-elected health providers have diverse personal reasons for wanting to engage in this role, and their engagement ebbs and flows with personal challenges and motivation levels. Local churches, non-profits, or entrepreneurial endeavours financially support a very limited numbers of CHW groups.

3. Incentives and business models: CHW roles vary from community to community with each CHW operating within that community's system. Incentives to work are based largely on the community, and while their jobs entitle them access to certain tools such as a weighing scale, simple drugs, or a glucometer, not all are 
afforded these benefits (Buehler at al, 2013). Due to their knowledge and access within the community, the CHW system sets the stage for business models for future healthcare systems and ultimately bridging the barrier between doctors and patients.

\section{DESIGNING RUGGEDISED DEVICES}

Solutions to poorly-engineered biomedical devices have been developed after four years of experiences designing, prototyping, and field testing in Kenya. The solutions presented in this section, which focus directly on the prototyping phase of the biomedical device design, have not been independently studied in a laboratory environment nor are they intended to be used on a mass-production scale where more advanced manufacturing techniques are available. Rather, these are in-field improvisations and observations that have emerged from extensive field experience in Kenya. These suggestions can be classified under the three larger categories of vibration and wire strain, dust and water penetration, and device usability. Engineers engaged in field-testing biomedical devices or other electronic appliances in resource-constrained environments will specifically benefit from these insights.

\subsection{Vibrations and wire strain}

Given the mobility requirements, biomedical devices must withstand regular vibrations from transport on unpaved roads as well as tugging on wires during the process of packing and unpacking instruments. Several solutions are presented to keep these shocks and strains from affecting sensors and damaging wires.

1. Vibrations-Secure sensitive elements in sealed plastic casings.

Pros: The plastic absorbs any shock and prevents fragile parts of the sensor from being exposed to high pressures. As shown in Figure 2 (section 3.4), a sensor can be secured in place by screwing the sensor to the housing so that the plastic can absorb stresses prior to their impact on the sensor.

Cons: Plastic casings add to the cost and can be lost if detachable pieces are not tied to the device.

2. Vibrations - Secure devices and spare parts in insulated carrying cases.

Pros: The case will absorb any shock experienced during travel through bumpy terrain. Insulation can be fashioned inexpensively using crumpled newspapers.

Cons: If not tightly secured in the travelling case, the packing material might move around and affect the quality of protection.

3. Wires - Tie knots or braid them together.

Pros: Tying knots before the connection points diverts the strain from the connection point to the knot itself, reducing the likelihood of signal corruption or disconnected wires. Similarly, braiding multiple wires together better distributes any mechanical loads on them.

Cons: Excessive strain in the knot may break the wire or disconnect wires from the sensor. Braiding wires together makes replacing individual wires more difficult.

4. Wires - Bind together with heat shrink reinforcement.

Pros: Heat shrinking provides extra reinforcement on wires and brings multiple wires into a single, stronger unit.

Cons: Finding and repairing broken wires can be more difficult within heat shrink, and replacing any removed wrap adds to maintenance costs.

5. Wires - Lengthen or lower wire gauge.

Pros: Longer wires allow the devices to have a broader range of movement to take into account the different patients' anthropometric requirements or different device positions. Lowering the gauge makes wires more impervious to bending and breaking.

Cons: Both options raise cost. Also, longer wires increase slack that could catch on something in transport, and lower gauge wires may be more difficult to bend or otherwise integrate into larger systems.

\subsection{Dust \& water penetration}

It is important that all devices are waterproof and dustproof, as exposure will cause damage to parts that are difficult or impossible to replace. Water damage can impact sensor accuracy as well as computer and DAQ operation. Dust can penetrate seals, degrade electrical circuits, clog filters, interfere with moving parts and damage surfaces.

\section{Test for potential of water damage}

Pros: Development-stage testing for resistance to both prolonged humidity and occasional rainstorm exposure mimics the rainy season conditions in tropical climates, allowing designers to identify problems before production.

Cons: Testing prolongs the design stage and may also raise development cost.

2. Cover sensors with uniform plastic film, wrapping or casing

Pros: Plastic is longer lasting, able to resist unsanitary substances, and easily shapeable. In addition, it will not degrade or rust the way wood or steel does.

Cons: Plastic may not be the most viable or inexpensive option for certain larger sensors, and may make troubleshooting and repairing sensors more difficult.

3. Transport in a protective bag 

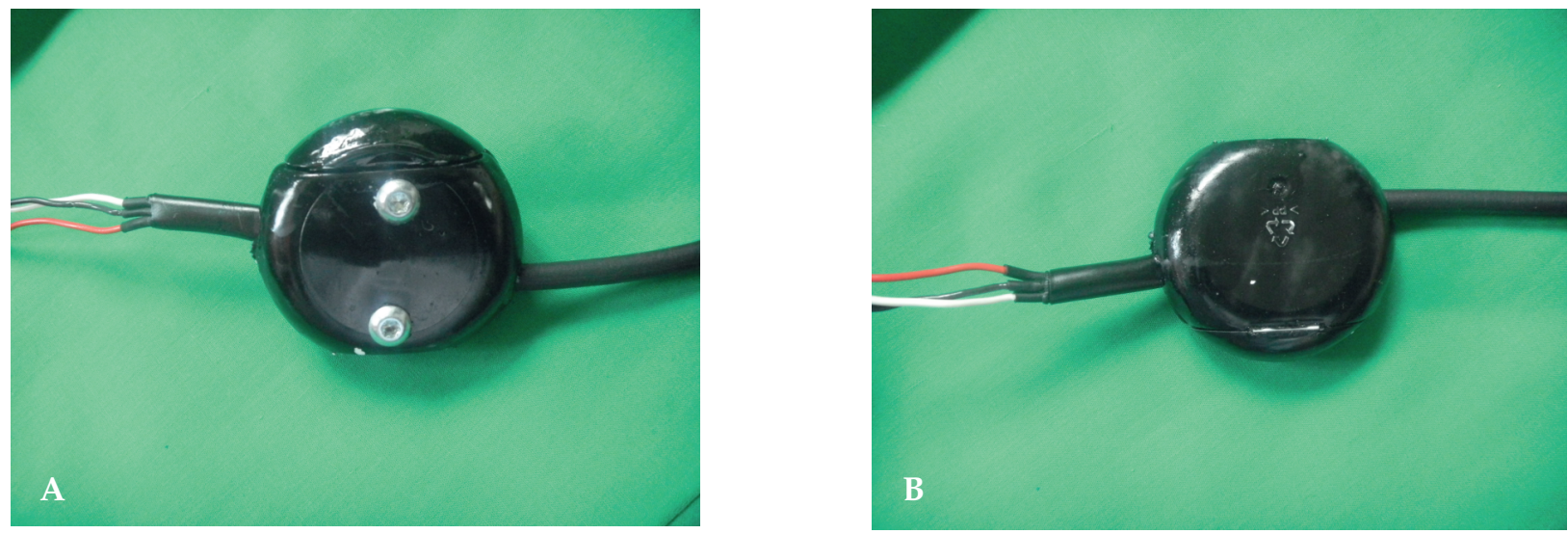

Figure 2: $\quad$ Figures 2A and 2B depict a strain relief mechanism through the sensor housing for a blood pressure monitor. Image A displays the first side on the housing, in which the sensor had been screwed into the walls for stability. Image B shows the reverse side of the sensor housing, which is uniform and completely sealed.

Pros: All-encompassing carrying bags can serve a triple purpose of transporting the devices, protecting from dust and rain, and organising the equipment.

Cons: Particularly in the rural operational context, many common homemade woven bags are not waterproof, and purchasing and maintaining a sufficiently usable bag adds to upkeep costs.

\section{Build with mild steel}

Pros: Mild steel is strong, easy to handle, and can be cleaned easily in addition to its abundant availability at a low cost.

Cons: Steel weighs more than wood or plastic options and lacks flexibility. Also, it is heavier and impacts the mobility of devices.

\section{Build with high-grade wood} and waterproof sealant

Pros: If wood is necessary, waterproofing will

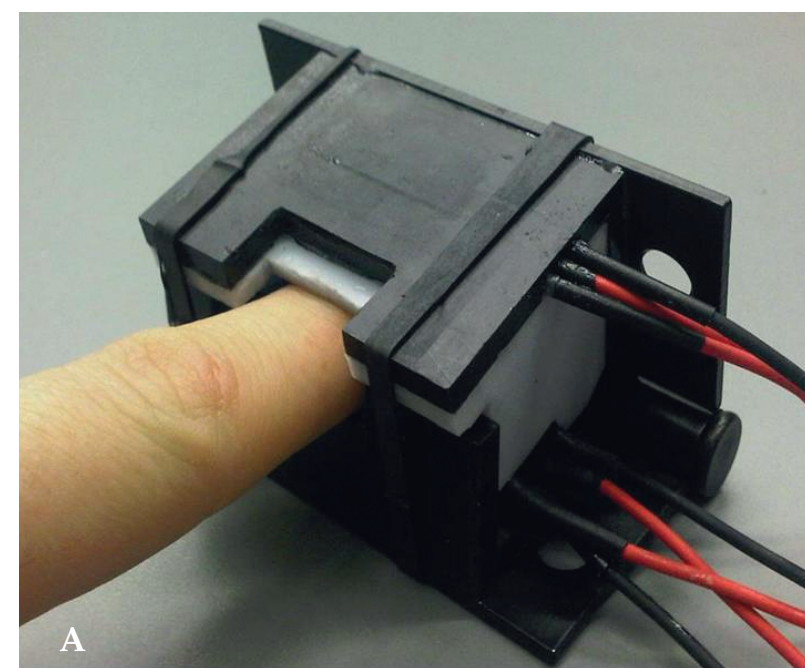

prolong its lifespan. It is easy to machine.

Cons: This raises both device and maintenance costs for wood that must be resealed yearly. It is not as strong as mild steel.

6. Clean regularly with water or rubbing alcohol

Pros: Cleaning keeps dust from causing surface deterioration, and rubbing alcohol also disinfects as needed.

Cons: Rubbing alcohol is not an environmentally sustainable solution and is an expensive consumable. Many areas have only limited access to clean water and might not have access to rubbing alcohol at all.

\subsection{Device usability}

The CHWs using the medical devices receive minimal training and typically lack formal education

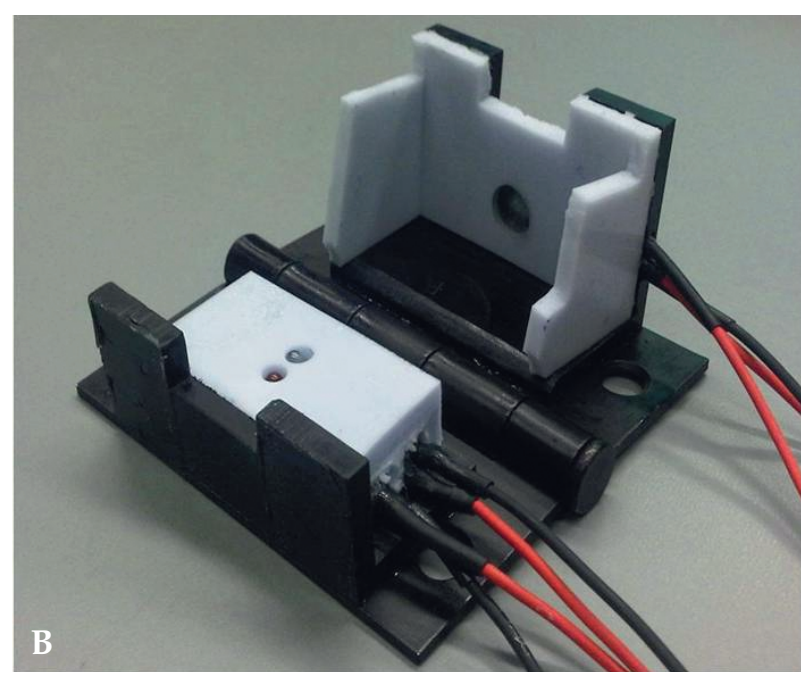

Figure 3: $\quad$ Figures 3A and 3B depict a pulse oximeter prototype. Image A shows a closed position during measurement, while Image B displays an open position. In both positions sensors are completely protected. 
in medicine or the use of biomedical devices. Biomedical instruments must be integrated into a system that an average CHW can easily and accurately operate. A few simple solutions enhance the user interface.

\section{Provide quick start guides and videos}

Pros: Visual guides that explain the device's operation in simple and logical steps can help reduce barriers to adoption. Laminated guides are more likely to survive than simple paper booklets. Video clips are the most effective media to educate CHWs on using the devices as it is a natural extension of their oral traditions. Guides should be developed in the official as well as local languages.

Cons: Though necessary, information about troubleshooting and maintenance increases length and complexity of these resources and is beyond the scope of what the CHWs can accomplish in the field. Unnecessary information might intimidate the CHWs.

\section{Interface with computers or smartphones}

Pros: Biomedical devices directly interfaced with the computer or smartphone reduces human error in recording readings.

Cons: Additional training is needed. The user interface must be simplified as much as possible.

\section{One-button programs}

Pros: Devices can be operated and understood with little training and regardless of technical background. Innovative technologies like the ForeRunner portable defibrillator have validated the efficacy of this concept (Forerunner for Heartstream, 2012).

Cons: The limited interface makes enabling additional functionality and troubleshooting more difficult.

\subsection{Lessons put to practice}

Penn State biomedical engineering students have employed solutions such as those listed above to create functional devices suitable for the rural context (Mathern et al, n.d.). While these concepts have been utilised through the design of ten different devices, the examples provided address many of the challenges that the resource-constrained environment poses. Simultaneously incorporating solutions to all of these challenges is difficult. Understanding user needs and balancing material costs with the requirements for ruggedness requires considerable analysis and creativity on the part of designers. Each unit comes with a simple quick start guide and video as supplemental material, and is operated by a onebutton program in LabVIEW. While employing the solutions described herein can help create functional devices, it is nearly impossible to design devices that will survive every challenge and solve every problem. The three examples provided in this section

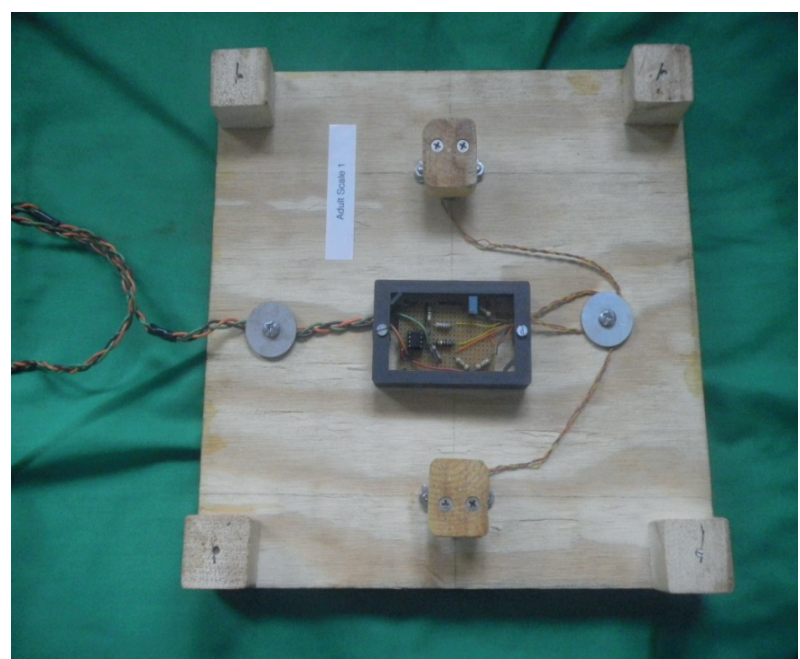

Figure 4: The underside of the adult scale employs several mechanisms to create a robust medical device including water and dust protection and strain relief techniques.

utilise multiple solutions to overcome the challenges of field-testing biomedical devices in a resourceconstrained context.

1. Blood pressure monitor - Different strain relief mechanisms have been used to secure the sensor for the blood pressure monitor as shown in Figure 2. Shrink wrapping has been used to reinforce high tension points in the wires as they exit the sensor housing. The sensor has been screwed to the inside of the plastic casing for stability, as the plastic will absorb stresses rather than the sensor itself. The entire sensor is completely sealed inside the walls of the plastic casing so that dust and water cannot penetrate it and damage the sensor or interrupt signals.

2. Pulse oximeter - A pulse oximeter such as the one shown in Figure 3 can measure heart rate and oxygen saturation in the blood. This ruggedised prototype frame has been built from mild steel and plastic to house the sensor. Heat shrink wrapping has been utilised at high stress points as the wires enter the sensor casing to provide additional reinforcement.

3. Adult weighing scale - A floor-standing adult scale that utilises strain gauges to measure a patient's weight. As shown in Figure 4, wires are secured to the underside of the scale using a washer, such that any wire strain is relegated to the knot rather than the strain gauges themselves. The excess wire between the scale and the DAQ is long and braided to accommodate greater distances while making the wire unit stronger. The circuit on the scale is encased in plastic to keep water and dust from affecting the sensor. The scale itself is made from high grade wood because this is a readily available, easy to manufacture, and able to withstand the force of 
body weight. The waterproof sealant that covers the wood protects it from dust penetration and water damage.

\section{CLOSING THOUGHTS}

Designing and field-testing biomedical devices for effective use in resource-constrained environments poses several challenges. The three multifaceted problems of vibration and wire strain, dust and water exposure and ease of use must be balanced against the fundamental constraints of capital and operational costs to yield ruggedised and durable biomedical devices. Several simple solutions towards this goal have been presented in this article. Additionally, leapfrogging solutions, which completely bypass the need for conventional biomedical devices can address these systemic challenges that make low-cost device design very challenging. For example, the emergence of smart phones has opened a market for potential healthcare solutions that do not need additional hardware. Mobile phone applications for wound care, capturing heart rhythms and informing nutrition are gaining popularity. Applications to measure vital signs directly from the smartphone would eliminate the need for external devices and further reduce costs and streamline the CHW's operations.

Despite the ubiquity of mobile phones in developing countries, smart phones are relatively rare and present a financial barrier to the uptake of highly virtualised instruments (Eulich, 2012). However, in recent years, several companies have been designing affordable smart phones for these emerging markets. Mozilla is launching Firefox Mobile OS phones in South American markets in 2013 (Keizer, 2012). Sales of less expensive smart phones are growing faster that high-end models in Western Europe and are considered crucial in emerging markets (World Bank, n.d.). In Kenya, a regular Android phone can be purchased for as little as US\$100 while an internet-enabled phone costs under US\$40. Although electricity is not always available within homes or workplaces, charged phones can be used during the day and then recharged at a central location during non-business hours. The CHWs' operational regimens can be designed to account for such contextspecific logistics.

Further, microfluidics and lab-on-chip systems offer compelling ways to reduce diagnostic costs. Microfluidic systems are being developed for largescale automation in chemistry and biology as well as chemical synthesis, screening compounds and preclinical testing of drugs in living cells (Dittrich \& Manz, 2006; Ricknas, 2011). For instance, a multi-channel microfluidic chip could use binding properties of proteins to identify certain diseases (Dittrich \& Manz, 2006). These chips can be interfaced with smartphones to yield point-of-care diagnostic and therapeutic systems. In essence, the rapid uptake of smart phones is clearly on the horizon and will be a 'game-changer' in the quest for ruggedised lowcost devices that $\mathrm{CHWs}$ can use in the field. Despite the immense potential of leapfrogging technologies, there remains a need for ruggedised devices in the near future. Since materials and enabling infrastructure to support the design, manufacturing and commercialisation of ruggedised biomedical devices are available, such engineering innovations can greatly enhance the efficiency and efficacy of Community Health Workers at the frontlines of global health.

\section{REFERENCES}

Buehler, B., Ruggiero, R. \& Mehta K. 2013, “Technology Opportunities for Enhancing the Effectiveness of Community Health Workers", IEEE Technology $\mathcal{E}$ Society Magazine, vol. 32, no. 1, Spring.

Chudi, Ibekwe 2010. "Healthcare Problems in Developing Countries". Medical Practice and Reviews April, Volume 1 ed.: pp. 9-11.

Dittrich, P. S. \& Manz, A. 2006, "Lab-on-a-chip: Microfluidics in Drug Discovery". Nature Reviews Drug Discovery 5.3: pp. 210-18.

Doctors of the World, Netherlands: Perspective, n.d., Ads of the World ${ }^{\mathrm{TM}}$. N.p., Web. 30 Nov. 2012. <http:/ / adsoftheworld.com/media/print/doctors_of_the_ world_netherlands_perspective?size=_original $>$.

Duke Global Health Institute, n.d., "About the Institute". N.p., Web. 27 Feb. 2013. http:// globalhealth.duke.edu/about-the-institute/

Dzombak, R., Mehta, K., \& Butler, P. 2012, “Design by Example: A Web-based Tool for Context-Driven Biomedical Device Design." NCIIA Open Conference, San Francisco, CA.

Eulich, W. 2012, "Developing Countries Lead the Way in Deploying Mobile Technology". The Christian Science Monitor, 28 July. Web. 30 Nov. <http: / / www. csmonitor.com/World/Global-Issues / 2012/0728/ Developing-countries-lead-the-way-in-deployingmobile-technology/\%28page\%29/2>.

Fleishman, A., Wittig, J., Milnes, J., Baxter, A., Moreau, J., Mehta, K. 2010, "Validation Process for a Social Entrepreneurial Venture in Tanzania: A Case Study", International Journal for Service Learning in Engineering: Humanitarian Engineering and Social Entrepreneurship, Vol 5, No 1.

"Forerunner for Heartstream", n.d., ForeRunner. N.p., Web. 12 Nov. 2012. <http://www.ideo.com/work/ forerunner $>$.

Foster, K. R. 2010, “Telehealth in Sub-Saharan Africa: Lessons for Humanitarian Engineering", IEEE Technology and Society Magazine, vol.29, no.1, pp. 42-49, Spring. 
"Johns Hopkins University Center for Bioengineering and Innovation Design", n.d., The Center for Bioengineering Innovation \& Design, Johns Hopkins University Whiting School of Engineering. N.p., Web. 27 Feb. 2013. http:/ / cbid.bme.jhu.edu/

Keizer, G. 2012, "Mozilla Promises Firefox OS Phones in Early 2013". Computerworld. N.p., 3 July. Web. 30 Nov. 2012. <http://www.computerworld.com/s / article/9228770/Mozilla_promises_Firefox_OS_ phones_in_early_2013>.

World Health Organisation, n.d. "Kenya”. N.p., Web. 12 Oct. 2012. <http:/ / www.who.int/countries/ken/ en $/>$.

Lekoubou A., Awah P., Fezeu L., Sobngwi E. \& Kengne A. P. 2010, Hypertension, Diabetes Mellitus and Task Shifting in Their Management in SubSaharan Africa. International Journal of Environmental Research and Public Health. 7, pp. 353-363.

Malkin, R. A. 2007, "Design of Health Care Technologies for the Developing World". Annual Review of Biomedical Engineering. 9: pp. 567-587.

Martin, J., Norris, B., Murphy, E. \& Crowe, J. 2008, "Medical Device Development: The Challenge for Ergonomics. Applied Ergonomics", 39 (3), pp. 271283.

Mathern, R., Schopman, S., Kalchthaler, K., Mehta, K. \& Butler, P. n.d., "Design of affordable and ruggedised biomedical devices using virtual instrumentation", Journal of Medical Engineering $\mathcal{E}$ Technology (In Press).
Peña-Mohr, J. 1987, “Distributing and Transferring Medical Technology". International Journal of Technology Assessment in Health Care, 3.02, p. 281.

Qin, R., Dzombak, R., Amin, R. \& Mehta, K. n.d., "Reliability of a Telemedicine System Designed for Rural Kenya", Journal of Primary Care and Community Health (In Press).

Ricknas, M. 2011, "Nokia Readies Linux OS for Low-end Smartphones". IT News, Technology Analysis and How-to Resources. N.p., 30 Sept. Web. 12 Nov. 2012. <http:/ / www.itworld.com/ hardware/208803/nokia-readies-linux-os-low-endsmartphones $>$.

United Nations Development Program, 2007, Human Development Report (HDR), November 27, p. 25.

World Bank, n.d., "Poverty Headcount Ratio at \$1.25 a Day (PPP) (\% of Population)". Data. N.p., Web. 30 Nov. 2012. <http:/ / data.worldbank.org/indicator/ SI.POV.DDAY>

World Health Organization, 2012. World Health Statistics 2012. Accessed December 16.

Young, F., Critchley, J. A., Johnstone, L. K. \& Unwin, N. C. 2009, A review of co-morbidity between infectious and chronic disease in Sub Saharan Africa: TB and Diabetes Mellitus, HIV and Metabolic Syndrome, and the impact of globalization. Globalization and Health; 5:9. 
